\title{
Supplementary Material for Dependent Species Sampling Models for Spatial Density Estimation
}

\author{
Seongil Jo*, Jaeyong Lee ${ }^{\dagger}$ Peter Müller ${ }^{\ddagger}$ Fernando A. Quintana ${ }^{\S}$ and Lorenzo Trippa
}

\section{Appendix A: Weak Supports of CAR SSM}

We prove that the CAR SSMs have full weak support. Having full weak support is one of the basic properties for any prior. We first state two general theorems of weak support for discrete probability measures which are of interest in their own right. Theorem 1 is for the case when both the weight and support processes are indexed by $x$.

Theorem 1. Let $\left(G_{x}=\sum_{h=1}^{\infty} p_{h}(x) \delta_{\theta_{h}(x)}, x \in \mathcal{X}\right)$ be a stochastic process of discrete probability measures on $\mathbb{R}$ indexed by $x \in \mathcal{X}$. Suppose the weight processes $\left(p_{h}(x), h \in \mathbb{N}_{+}, x \in \mathcal{X}\right)$ and support processes $\left(\theta_{h}(x), h \in \mathbb{N}, x \in \mathcal{X}\right)$ are independent. Let $S^{k}:=\left\{\left(p_{1}, \ldots, p_{k}\right): 0<\sum_{h=1}^{k} p_{h}<\right.$ 1 and $\left.p_{h}>0, h=1,2, \ldots, k\right\}$. If for any positive integers $n, k_{1}, k_{2}, \ldots, k_{n}$, and $x_{1}, x_{2}, \ldots, x_{n} \in \mathcal{X}$,

(i) $\left(\theta_{1}\left(x_{1}\right), \ldots, \theta_{k_{1}}\left(x_{1}\right) ; \theta_{1}\left(x_{2}\right) \ldots, \theta_{k_{2}}\left(x_{2}\right) ; \ldots ; \theta_{1}\left(x_{n}\right) \ldots, \theta_{k_{n}}\left(x_{n}\right)\right)$ has support $\mathbb{R}^{k_{1}} \times \mathbb{R}^{k_{2}} \times \ldots \times$ $\mathbb{R}^{k_{n}}$ and,

(ii) $\left(p_{1}\left(x_{1}\right), \ldots, p_{k_{1}}\left(x_{1}\right) ; p_{1}\left(x_{2}\right) \ldots, p_{k_{2}}\left(x_{2}\right) ; \ldots ; p_{1}\left(x_{n}\right) \ldots, p_{k_{n}}\left(x_{n}\right)\right)$ has support $S^{k_{1}} \times S^{k_{2}} \times \ldots \times$ $S^{k_{n}}$

then, $\left(G_{x}, x \in \mathcal{X}\right)$ has full weak support.

The following theorem is for the case when a single set of $\theta$ random variables are used as common support points for all the discrete probability measures.

Theorem 2. Let $\left(G_{x}=\sum_{h=1}^{\infty} p_{h}(x) \delta_{\theta_{h}}, x \in \mathcal{X}\right)$ be a stochastic process of discrete probability measures on $\mathbb{R}$ indexed by $x \in \mathcal{X}$. Suppose the weight processes $\left(p_{h}(x), i \in \mathbb{N}_{+}, x \in \mathcal{X}\right)$ and support points $\left(\theta_{h}, i \in \mathbb{N}\right)$ are independent. If for any positive integers $n, k$ and $x_{1}, x_{2}, \ldots, x_{n} \in \mathcal{X}$,

(i) $\left(\theta_{1}, \ldots, \theta_{k}\right)$ has support $\mathbb{R}^{k}$ and

(ii) $\left(p_{1}\left(x_{1}\right), \ldots, p_{k}\left(x_{1}\right) ; p_{1}\left(x_{2}\right) \ldots, p_{k}\left(x_{2}\right) ; \ldots ; p_{1}\left(x_{n}\right) \ldots, p_{k}\left(x_{n}\right)\right)$ has support $S^{k} \times S^{k} \times \ldots \times S^{k}$,

then, $\left(G_{x}, x \in \mathcal{X}\right)$ has full weak support.

It is easy to extend Theorems 1 and 2 , which assume $\theta_{i}\left(x_{j}\right) \in \mathbb{R}$, to the more general case of $\theta_{i}\left(x_{j}\right) \in \mathbb{R}^{d}$. In particular, in our application $\theta_{i}\left(x_{j}\right) \in \mathbb{R}^{2}$.

The main result of this subsection requires the following simple lemma whose proof is omitted.

Lemma 1. Suppose $f: \mathcal{X} \longrightarrow \mathcal{Y}$ is continuous and onto. If a random variable $X$ has full support, i.e. the distribution of $X$ has support $\mathcal{X}$, then $Y=f(X)$ has also full support.

Now we give the proof for Proposition 1 of Section 3 of the main paper.

Proof of Proposition 1. We prove the result for the Mercer CAR SSM. The proof for the CK CAR SSM is similar. We prove the claim by showing conditions (i) and (ii) of Theorem 2. Since $G_{0}$ has full support, (i) is satisfied. Note that the weight process $\left(p_{s, h}, s \in \mathcal{D}, h=1,2, \ldots\right)$ is defined by a continuous map from the autoregressive process $\left(u_{s, h}, s \in \mathcal{D}, h=1,2, \ldots\right)$. Since for each $h$,

\footnotetext{
*Department of Statistics, Korea University, Republic of Korea, joseongil@gmail.com

$\dagger$ Department of Statistics, Seoul National University, Republic of Korea, leejyc@gmail.com

$\ddagger$ Department of Mathematics, University of Texas at Austin, USA, pmueller@math.utexas.edu

$\S$ Departamento de Estadísica, Pontificia Universidad Católica de Chile, Chile, quintana@mat.uc.cl

๑Department of Biostatistics, Dana-Farber Cancer Institute and Havard University, USA, ltrippa@jimmy.harvard.edu
} 
$\left(u_{s, h}, s \in \mathcal{D}\right)$ follows a nonsingular normal distribution and has full support on $\mathbb{R}^{n}$ and also for all $h,\left(u_{s, h}, s \in \mathcal{D}\right)$ are independent of $\left(u_{s, h^{\prime}}, s \in \mathcal{D}, h^{\prime} \neq h\right),\left(u_{s, h}, s \in \mathcal{D}, h=1,2, \ldots\right)$ has full support. Thus, by Lemma 1, condition (ii) of Theorem 1 is satisfied.

\section{A.1 Proof of Theorem 1}

Let $\mathcal{F}$ be the space of all probability measures on $\mathbb{R}$ with finite supports and $C$ be the set of all bounded continuous functions on $\mathbb{R}$. By Theorem II.6.3 of Parthasarathy (1967), $\mathcal{F}$ is dense in the space of all probability measures on $\mathbb{R}$ under the weak topology. To show that $\left(G_{x}, x \in \mathcal{X}\right)$ has the full weak support, we need to show that for any given $\epsilon>0$, positive integers $n, k_{1}, \ldots, k_{n}$, $x_{1}, x_{2}, \ldots, x_{n} \in \mathcal{X}$, probability measures with finite supports $G_{x_{i}}^{0}=\sum_{l=1}^{k_{i}} p_{i l}^{0} \delta_{\theta_{i l}^{0}}, i=1,2, \ldots, n$ and $f_{i j} \in C$ with $i=1,2, \ldots, n$ and $j=1, \ldots, m_{i}$, the basic open set

$$
U=\left\{\left(G_{x}, x \in \mathcal{X}\right):\left|\int f_{i j} d G_{i}-\int f_{i j} d G_{i}^{0}\right|<\epsilon, i=1,2, \ldots, n, j=1,2, \ldots, m_{i}\right\}
$$

has positive probability.

Let $M=\sup _{i, j} \sup _{\theta}\left|f_{i j}(\theta)\right|$ and $k=\max _{i} k_{i}$. Since $f_{i j}$ are continuous, we can choose $\delta>0$ so that $\left|\theta-\theta_{i l}^{0}\right|<\delta$ implies $\left|f_{i j}(\theta)-f_{i j}\left(\theta_{i l}^{0}\right)\right|<\epsilon / 3$ for all $i, j, l$. Let

$$
\begin{aligned}
V_{w} & :=\left\{\left(p_{11}, \ldots, p_{1, k_{1}} ; \ldots ; p_{n, 1}, \ldots, p_{n, k_{n}}\right):\left|p_{i l}-p_{i l}^{0}\right|<\frac{\epsilon}{3 M k}, i=1,2, \ldots, n, l=1,2, \ldots, k_{i}\right\} \\
V_{s} & :=\left\{\left(\theta_{11}, \ldots, \theta_{1, k_{1}} ; \ldots ; \theta_{n 1}, \ldots, \theta_{n, k_{n}}\right):\left|\theta_{i l}-\theta_{i l}^{0}\right|<\delta, i=1,2, \ldots, n, l=1,2, \ldots, k_{i}\right\} .
\end{aligned}
$$

By the assumptions, $V_{w} \times V_{s}$ has positive probability. We will complete the proof by showing that $\left(G_{x}, x \in \mathcal{X}\right)$ with weights in $V_{w}$ and supports in $V_{s}$ belongs $U$. For all $i$ and $j$,

$$
\begin{gathered}
\left|\int f_{i j} d G_{i}(\theta)-\int f_{i j} d G_{i}^{0}(\theta)\right|=\left|\sum_{l=1}^{\infty} p_{i l} f_{i j}\left(\theta_{l}\right)-\sum_{l=1}^{k_{i}} p_{i l}^{0} f_{i j}\left(\theta_{l}^{0}\right)\right| \\
\leq \sum_{l=1}^{k_{i}}\left|p_{i l} f_{i j}\left(\theta_{l}\right)-p_{i l}^{0} f_{i j}\left(\theta_{l}^{0}\right)\right|+\sum_{l=k_{i}}^{\infty} p_{i l}\left|f_{i j}\left(\theta_{l}\right)\right| \\
\leq \sum_{l=1}^{k_{i}} p_{i l}\left|f_{i j}\left(\theta_{l}\right)-f_{i j}\left(\theta_{l}^{0}\right)\right|+\sum_{l=1}^{k_{i}}\left|f_{i j}\left(\theta_{l}^{0}\right)\right| \cdot\left|p_{i l}-p_{i l}^{0}\right|+\sum_{l=k_{i}}^{\infty} p_{i l}\left|f_{i j}\left(\theta_{l}\right)\right| \\
<\sum_{l=1}^{k_{i}} p_{i l} \frac{\epsilon}{3}+M k \frac{\epsilon}{3 M k}+M \frac{\epsilon}{3 M} \leq \epsilon
\end{gathered}
$$

The last strict inequality is due to the following inequalities

$$
\sum_{l=k_{i}+1}^{\infty} p_{i l}=\left|\left(1-\sum_{l=1}^{k_{i}} p_{i l}\right)-\left(1-\sum_{l=1}^{k_{i}} p_{i l}^{0}\right)\right| \leq \sum_{l=1}^{k_{i}}\left|p_{i l}-p_{i l}^{0}\right| \leq k_{i} \frac{\epsilon}{3 M k} \leq \frac{\epsilon}{3 M} .
$$

This completes the proof.

\section{A.2 Proof of Theorem 2}

As in the proof of Theorem 1, we will prove that for a given $\epsilon>0$, positive integers $n, k_{1}, \ldots, k_{n}$, $x_{1}, x_{2}, \ldots, x_{n} \in \mathcal{X}$, probability measures with finite supports $G_{x_{i}}^{0}=\sum_{l=1}^{k_{i}} p_{i l}^{0} \delta_{\theta_{i l}^{0}}, i=1,2, \ldots, n$ and $f_{i j} \in C$ with $i=1,2, \ldots, n$ and $j=1, \ldots, m_{i}$, the basic open set

$$
U=\left\{\left(G_{x}, x \in \mathcal{X}\right):\left|\int f_{i j} d G_{i}-\int f_{i j} d G_{i}^{0}\right|<\epsilon, i=1,2, \ldots, n, j=1,2, \ldots, m_{i}\right\}
$$

has positive probability. 
Let $\left\{\theta_{1}^{0}, \theta_{2}^{0}, \ldots, \theta_{k}^{0}\right\}=\cup_{i=1}^{n}\left\{\theta_{i l}^{0}: l=1,2, \ldots, k_{i}\right\}$ with cardinality $k$. We can represent $G_{x_{i}}^{0}$ with new support points $G_{x_{i}}^{0}=\sum_{l=1}^{k} p_{i l}^{*} \delta_{\theta_{l}^{0}}$ with $p_{i l}^{*}=0$ if $\theta_{l}$ does not belong to the support of $G_{x_{i}}^{0}$. Let

$$
\begin{aligned}
V_{w} & :=\left\{\left(p_{11}, \ldots, p_{1, k} ; \ldots ; p_{n, 1}, \ldots, p_{n, k}\right):\left|p_{i l}-p_{i l}^{*}\right|<\delta_{1}, i=1,2, \ldots, n, l=1,2, \ldots, k\right\} \\
V_{s} & :=\left\{\left(\theta_{11}, \ldots, \theta_{1, k}\right):\left|\theta_{i l}-\theta_{i l}^{0}\right|<\delta_{2}, i=1,2, \ldots, n, l=1,2, \ldots, k_{i}\right\} .
\end{aligned}
$$

By the assumptions, $V_{w} \times V_{s}$ has positive prior probability. Similarly to the proof of Theorem 1, one can show that that $\left(G_{x}, x \in \mathcal{X}\right)$ with weights in $V_{w}$ and supports in $V_{s}$ belongs $U$. This completes the proof.

\section{Appendix B: Full conditionals and MCMC steps}

The joint posterior density derived from the hierarchical model (4.5) of Section 4.1 of the main paper is

$$
\begin{aligned}
\pi\left(\boldsymbol{\mu}, \boldsymbol{\sigma}^{2}, \boldsymbol{u}, \boldsymbol{z}, \mu_{0}, \alpha, \psi \mid \boldsymbol{y}\right) \propto & \prod_{i=1}^{n} \prod_{j=1}^{N_{i}}\left\{N\left(y_{i j} ; \mu_{z_{i j}}, \sigma_{z_{i j}}^{2}\right)\right\} \prod_{i=1}^{n} \prod_{j=1}^{N_{i}}\left(\frac{e^{u_{i z_{i j}}}}{\sum_{k=1}^{K} e^{u_{i k}}}\right) \times \\
& \times \prod_{h=1}^{K} p\left(u_{1 h}, \ldots, u_{n h} \mid \eta\right) \prod_{h=1}^{K} N\left(\mu_{h} ; \mu_{0}, \sigma_{h}^{2} / \alpha\right) G a\left(\sigma_{h}^{-2} ; \frac{\nu}{2}, \frac{\nu \psi}{2}\right),
\end{aligned}
$$

where $\eta=\left(a, b, \tau^{2}, \rho\right), \mu_{0}, \in \mathbb{R}$ and $\alpha, \nu, \psi>0$. We implement posterior inference by Markov chain Monte Carlo posterior simulation using the following transition probabilities. We start by sampling $z_{i j} \sim p\left(z_{i j} \mid\right.$ rest $)$ and $\left(\mu_{h}, \sigma_{h}^{2} \mid\right.$ rest $)$. The notation $p(\cdot \mid$ rest $)$ is used to indicate full conditional distributions used in Gibbs-type transitions. Both are straightforward standard complete conditional posterior distribution. Similarly, $\mu_{0}, \alpha$ and $\psi$ are updated by draws from the complete conditional posterior which are given below.

- For each $i, j$,

$$
\operatorname{Pr}\left(z_{i j}=h \mid \text { rest }\right) \propto P_{i h} N\left(y_{i j} \mid \mu_{h}, \sigma_{h}^{2}\right), \quad h=1, \ldots, K,
$$

where $P_{i h}=e^{u_{i h}} / \sum_{k=1}^{K} e^{u_{i k}}$

- For each $h=1, \ldots, K$

$$
\begin{aligned}
\pi\left(\mu_{h}, \sigma_{h}^{-2} \mid \text { rest }\right) \propto N\left(\mu_{h} ; \mu_{0}, \sigma_{h}^{2} / \alpha\right) G a\left(\sigma_{h}^{-2} ; \frac{\nu}{2}, \frac{\nu \psi}{2}\right) & \prod_{\left\{(i, j): z_{i j}=h\right\}} N\left(y_{i j} ; \mu_{h}, \sigma_{h}^{2}\right) \\
& \propto N\left(\mu_{h} ; \mu_{h}^{*}, \sigma_{h}^{2} / \alpha_{h}^{*}\right) G a\left(\sigma_{h}^{-2} ; \frac{\nu_{h}^{*}}{2}, \frac{\nu_{h}^{*} \psi_{h}^{*}}{2}\right),
\end{aligned}
$$

where $n_{h}=\sum_{i=1}^{n} \sum_{j=1}^{N_{i}} I\left(z_{i j}=h\right), \bar{y}_{h}=\frac{1}{n_{h}} \sum_{\left\{(i, j): z_{i j}=h\right\}} y_{i j}, s_{h}^{2}=\frac{1}{n_{h}-1} \sum\left(y_{i j}-\bar{y}_{h}\right)^{2}$, and

$$
\mu_{h}^{*}=\frac{n_{h} \bar{y}_{h}+\alpha \mu_{0}}{n_{h}+\alpha}, \alpha_{h}^{*}=n_{h}+\alpha, \nu_{h}^{*}=\nu+n_{h}, \psi^{*}=\frac{\nu \psi+\left(n_{h}-1\right) s_{h}^{2}+\frac{\alpha n_{h}}{\alpha_{h}^{*}}\left(\bar{y}_{h}-\mu_{0}\right)^{2}}{\nu_{h}^{*}} .
$$

- For each $i$, the full conditional distribution for the weights $\boldsymbol{u}_{i}=\left(u_{i, h}, h=1, \ldots, K\right)$, is

$$
\begin{aligned}
\pi\left(\boldsymbol{u}_{i} \mid \text { rest }\right) \propto \prod_{j=1}^{N_{i}} \frac{e^{u_{i, z_{i j}}}}{\sum_{k} e^{u_{i, k}}} & \prod_{h=1}^{K} p\left(u_{i, h} \mid u_{\ell, h}, \ell \neq i, \eta\right) \\
& =\prod_{j=1}^{N_{i}} \frac{e^{u_{i z_{i j}}}}{\sum_{k} e^{u_{i k}}} \prod_{h=1}^{K} N\left(u_{i, h} ; m_{i, h}-\sum_{\ell: \ell \neq i}^{n} \xi_{i \ell}\left(u_{\ell, h}-m_{\ell, h}\right), \tau^{2}\right) .
\end{aligned}
$$


To sample $\boldsymbol{u}_{i}$, we introduce latent variables $\boldsymbol{\lambda}=\left(\lambda_{i j h}\right)$, with $\lambda_{i j h} \sim \operatorname{Exp}\left(e^{u_{i, h}}\right), i=1, \ldots, n$, $j=1, \ldots, N_{i}, h=1, \ldots, K$, where $\operatorname{Exp}(d)$ denotes the exponential distribution with mean $1 / d$. Then, $\operatorname{argmin}_{k}\left\{\lambda_{i j k}\right\}$ has the same distribution as $z_{i j}$, that is,

$$
\operatorname{Pr}\left(\lambda_{i j h}=\min _{k} \lambda_{i j k}\right)=\frac{e^{u_{i, h}}}{\sum_{k} e^{u_{i, k}}}=\operatorname{Pr}\left(z_{i j}=h \mid \text { rest }\right) .
$$

Using this exponential race, we redefine $z_{i j}=\operatorname{argmin}_{k} \lambda_{i j k}$. Also, let $\operatorname{Exp}(x \mid d)$ denote an exponential p.d.f. with mean $1 / d$, evaluated at $x$. Let $R_{i j}=\left\{\boldsymbol{\lambda}: \lambda_{i j z_{i j}}<\lambda_{i j h}, h \neq z_{i j}\right\}$ denote the event that $\lambda_{i j z_{i j}}$ wins the exponential race. Then, we have

$$
\begin{aligned}
\pi\left(\boldsymbol{u}_{i} \mid \text { rest }\right) & \propto \prod_{j=1}^{N_{i}}\left(\frac{e^{u_{i, z_{i j}}}}{\sum_{k} e^{u_{i, k}}}\right) \prod_{h=1}^{K} N\left(u_{i, h} ; m_{i, h}-\sum_{\ell: \ell \neq i}^{n} \xi_{i \ell}\left(u_{\ell, h}-m_{\ell, h}\right), \tau^{2}\right) \\
= & \prod_{j=1}^{N_{i}}\left\{\int_{R_{i j}} \prod_{k=1}^{K} \operatorname{Exp}\left(\lambda_{i j k} \mid e^{u_{i, k}}\right) d \boldsymbol{\lambda}\right\} \prod_{h=1}^{K} N\left(u_{i, h} ; m_{i, h}-\sum_{\ell: \ell \neq i}^{n} \xi_{i \ell}\left(u_{\ell, h}-m_{\ell, h}\right), \tau^{2}\right)
\end{aligned}
$$

and

$$
\begin{aligned}
\pi\left(\boldsymbol{u}_{i}, \boldsymbol{\lambda}_{i} \mid \text { rest }\right) \propto \prod_{j=1}^{N_{i}}\left\{\prod_{h=1}^{K} \operatorname{Exp}\left(\lambda_{i j h} \mid e^{u_{i, h}}\right) I_{R_{i j}}(\boldsymbol{\lambda})\right\} \\
\\
\quad \times \prod_{h=1}^{K} N\left(u_{i, h} ; m_{i, h}-\sum_{\ell: \ell \neq i}^{n} \xi_{i \ell}\left(u_{\ell, h}-m_{\ell, h}\right), \tau^{2}\right),
\end{aligned}
$$

where $\boldsymbol{\lambda}_{i}=\left(\lambda_{i j h}, j=1, \ldots, N_{i}, h=1, \ldots, K\right)$.

The sampling step for $\boldsymbol{u}_{i}$ thus consists of iteratively sampling $\boldsymbol{u}_{i}$ and $\boldsymbol{\lambda}_{i}$. We first generate $\lambda_{i j, z_{i j}} \sim \operatorname{Exp}\left(\sum_{k=1}^{K} e^{u_{i, k}}\right)$, and then, exploiting the memoryless property of the exponential distribution, $\lambda_{i j h}=\lambda_{i j, z_{i j}}+\lambda_{i j h}^{*}$, with $\lambda_{i j h}^{*} \sim \operatorname{Exp}\left(e^{u_{i, h}}\right), h \neq z_{i j}$. Finally, conditional on the latent variables, $\boldsymbol{\lambda}_{i}$, we generate $u_{i, h}, h=1, \ldots, K$, from

$$
\begin{aligned}
\pi\left(u_{i, h} \mid \text { rest }\right) & \propto N\left(u_{i, h} ; m_{i h}-\sum_{\ell: \ell \neq i}^{n} \xi_{i \ell}\left(u_{\ell, h}-m_{\ell h}\right), \tau^{2}\right) \prod_{j=1}^{N_{i}} e^{u_{i, h}} e^{-\lambda_{i j h} e^{u_{i, h}}} \\
& \propto e^{-\frac{1}{2 \tau^{2}}\left(u_{i, h}-m_{i h}+\sum_{\ell: \ell \neq i}^{n} \xi_{i \ell}\left(u_{\ell, h}-m_{\ell h}\right)\right)^{2}} \prod_{j=1}^{N_{i}} e^{u_{i, h}} e^{-\lambda_{i j h} e^{u_{i, h}}} .
\end{aligned}
$$

Unfortunately, this expression does not correspond to any known distribution. We draw $u_{i, h}$ using an adaptive Metropolis within Gibbs algorithm presented by Roberts and Rosenthal (2009).

\section{References}

Parthasarathy, K. R. (1967). Probability measures on metric spaces. Probability and Mathematical Statistics, No. 3. Academic Press, Inc., New York-London.

Roberts, G. O. and Rosenthal, J. S. (2009). "Examples of adaptive MCMC." J. Comput. Graph. Statist., 18(2): 349-367. 4 